\title{
Hollow sphere as a detector of gravitational radiation
}

\author{
E. Coccia \\ Dipartimento di Fisica, Università di Roma "Tor Vergata”' and INFN Sezione di Roma Tor Vergata, Via Ricerca Scientifica 1, \\ 00133 Roma, Italy \\ V. Fafone \\ INFN Laboratori Nazionali di Frascati, Via E. Fermi 40, 00044 Frascati, (Roma), Italy \\ G. Frossati \\ Kamerlingh Onnes Laboratory, Leiden University, Leiden, The Netherlands \\ J. A. Lobo and J. A. Ortega \\ Departament de Física Fonamental, Universitat de Barcelona, Spain \\ (Received 6 June 1997; published 14 January 1998)
}

\begin{abstract}
The most important features of the proposed spherical gravitational wave detectors are closely linked with their symmetry. Hollow spheres share this property with solid ones, considered in the literature so far, and constitute an interesting alternative for the realization of an omnidirectional gravitational wave detector. In this paper we address the problem of how a hollow elastic sphere interacts with an incoming gravitational wave and find an analytical solution for its normal mode spectrum and response, as well as for its energy absorption cross sections. It appears that this shape can be designed having relatively low resonance frequencies $(\sim 200 \mathrm{~Hz})$ yet keeping a large cross section, so its frequency range overlaps with the projected large interferometers. We also apply the obtained results to discuss the performance of a hollow sphere as a detector for a variety of gravitational wave signals. [S0556-2821(97)00522-5]
\end{abstract}

PACS number(s): 04.80.Nn, 95.55.Ym

\section{INTRODUCTION}

Thirty-five years after the beginning of the experimental search for cosmic gravitational waves (GW's), several resonant-mass detectors (cryogenic cylindrical bars) are currently monitoring the strongest potential sources in our Galaxy and in the local group [1]. The sensitivity of such detectors is $h \simeq 6 \times 10^{-19}$ for millisecond GW bursts or, in spectral units, $10^{-21} \mathrm{~Hz}^{-1 / 2}$ over a bandwidth of a few $\mathrm{Hz}$ around $1 \mathrm{kHz}$. A further improvement in sensitivity and bandwidth is expected from the operation at ultralow temperatures of the two bar detectors NAUTILUS [2] and AURIGA [3] in Italy, and even better sensitivities and bandwidths will come about as more advanced readout systems are developed. Projects for spherical resonant-mass GW detectors have emerged in the last few years in the resonantmass community [4-7], due to their remarkable advantages with respect to the operating bars [7].

In a cylindrical bar only the first longitudinal mode of vibration interacts strongly with the $\mathrm{GW}$, and consequently only one wave parameter can be measured: the amplitude of a combination of the two polarization states. On the other hand each quadrupole mode of a spherical mass is fivefold degenerate [its angular dependence is described in terms of the five spherical harmonics $Y_{l m}(\theta, \varphi)$ with $l=2$ and $m=-2, \ldots, 2]$, and presents an isotropic cross section. The cross section of the lowest order $(n=1)$ mode is the highest, and is larger than that of a cylindrical antenna made of the same material and with the same resonant frequency by a factor of about $0.8\left(R_{s} / R_{b}\right)^{2}[6,7]$, where $R_{s}$ and $R_{b}$ are the radius of the sphere and of the bar, respectively. This means a factor of 20 over present bars. Moreover, the sphere's cross section is also high at its second quadrupole harmonic.

The fivefold degeneracy of the quadrupole modes enables the determination of the GW amplitudes of two polarization states and the two angles of the source direction. The method, first outlined by Forward [9] and later developed by Wagoner and Paik [10], consists in measuring the sphere vibrations in at least five independent locations on the sphere surface so as to determine the vibration amplitude of each of the five degenerate modes. The Fourier components of the GW amplitudes at any quadrupole frequencies and the two angles defining the source direction can be obtained as suitable combinations of these five outputs $[5,6,8,11,12]$.

The signal deconvolution is based on the assumption that in the wave frame (that in which the $z$ axis is aligned with the wave propagation direction) only the $l=2$ and $m= \pm 2$ modes are excited by the GW, as the helicity of a GW is 2 in general relativity. One can take advantage of this to deconvolve the wave propagation direction and the GW amplitudes in the wave frame.

Most of the nice properties of a spherical GW detector depend on its being spherically symmetric. A spherical shell, or hollow sphere, obviously maintains that symmetry, and so it can be considered an interesting alternative to the usual solid sphere. In order to have a good cross section, a resonant GW detector must be made of a high speed of sound material and have a large mass. The actual construction of a massive spherical body may be technically difficult. In fact, fabricating a large hollow sphere is a different task than fabricating a solid one. Casting a hollow half sphere is a nearly twodimensional cast, at odds with casting a solid sphere, which 
requires rather special moulds. As an example of the feasibility of large two-dimensional casting we can mention the fabrication of propellers of more than $10 \mathrm{~m}$ in size and masses of the order of 100 tons [13]. Two hollow hemispheres could then be welded together with electron beam techniques. However, while it is known that these welding technique preserve most of the properties of the bare material, its effect on the acoustic quality factor (a relevant paramenter in resonant mass detectors) must be further studied.

We have investigated the properties of a hollow sphere as a potential GW antenna. The purpose of this paper is to present a detailed report of the main results of such an investigation and to discuss the real interest of this new detector shape.

In Sec. II we present the complete analytical solution of the eigenmode problem for a hollow sphere of arbitrary thickness, including the full frequency and amplitude spectrum. Section III is devoted to the cross section analysis, while in Sec. IV we take up the study of the system sensitivity to various $\mathrm{GW}$ signal classes. Finally, we present an outlook and summary of conclusions in Sec. V.

\section{NORMAL MODES OF VIBRATION AND EIGENFREQUENCIES OF A HOLLOW SPHERE}

In this section we consider the problem of a hollow elastic sphere in order to obtain its normal modes and frequency spectrum. This is a classical problem in elasticity theory which was posed and partly addressed already in the last century; see, e.g., [14] and references therein.

Let $R$ and $a$ be the outer and inner radii of the sphere, respectively. The elastic properties of the sphere, provided it is homogenous and isotropic, will be described by its Lamè coefficients $\lambda$ and $\mu$ and its density $\rho$. As is well known (see, e.g., [8]), the normal modes are obtained as the solutions to the eigenvalue equation

$$
\nabla^{2} \mathbf{u}+(1+\lambda / \mu) \nabla(\nabla \cdot \mathbf{u})=-k^{2} \mathbf{u} \quad\left(k^{2} \equiv \omega^{2} \rho / \mu\right),
$$

subject to the boundary conditions that the solid's surface be free of any tensions and/or tractions; these are expressed by the equations

$$
\sigma_{i j} n_{j}=0 \quad \text { at } \quad r=R \quad \text { and at } \quad r=A \quad(R \geqslant a \geqslant 0),
$$

where the sphere's surface $S$ has outward normal $\mathbf{n}$. The possibility of a spherical shell $(a=R)$ and that of a solid sphere $(a=0)$ are allowed. The stress tensor $\sigma_{i j}$ is given by [8]

$$
\sigma_{i j}=\lambda u_{k, k} \delta_{i j}+2 \mu u_{(i, j)} .
$$

The general solution to Eq. (2.1) can be cast in the form

$$
\begin{aligned}
\mathbf{u}(\mathbf{x})= & C_{0} \nabla \phi(\mathbf{x})+i C_{1} \mathbf{L} \psi(\mathbf{x})+i C_{2} \nabla \times \mathbf{L} \psi(\mathbf{x})+D_{0} \nabla \widetilde{\phi}(\mathbf{x}) \\
& +i D_{1} \mathbf{L} \widetilde{\psi}(\mathbf{x})+i D_{2} \nabla \times \mathbf{L} \widetilde{\psi}(\mathbf{x}),
\end{aligned}
$$

where $C_{i}$ and $D_{i}$ are constants, $\mathbf{L} \equiv-i \mathbf{x} \times \nabla$, and the scalar functions $\phi, \psi, \widetilde{\phi}$, and $\widetilde{\psi}$ are given by

$$
\phi(\mathbf{x})=j_{l}(q r) Y_{l m}(\theta, \varphi), \quad \psi(\mathbf{x})=j_{l}(k r) Y_{l m}(\theta, \varphi)
$$

$$
\widetilde{\phi}(\mathbf{x})=y_{l}(q r) Y_{l m}(\theta, \varphi), \quad \widetilde{\psi}(\mathbf{x})=y_{l}(k r) Y_{l m}(\theta, \varphi),
$$

where $q \equiv k \sqrt{\mu /(\lambda+\mu)}$ and $Y_{l m}$ denotes a spherical harmonic. Finally, $j_{l}$ and $y_{l}$ are the standard Bessel functions of the first and second kinds, respectively (see, e.g., [15]). The latter (which are singular at the origin) must be included in our case, as $r=0$ lies outside the boundary $S$. The boundary conditions (2.2) become, after rather lengthy calculations, a system of linear equations which splits up into a $4 \times 4$ linear system for $\left(C_{0}, C_{2}, D_{0}, D_{2}\right)$ and a $2 \times 2$ system for $\left(C_{1}, D_{1}\right)$. That is, we have a linear system of the form

$$
\left(\begin{array}{cc}
\mathbf{A}_{\mathbf{P}} & 0 \\
0 & \mathbf{A}_{\mathbf{T}}
\end{array}\right)\left(\begin{array}{l}
\mathbf{C}_{\mathbf{P}} \\
\mathbf{C}_{\mathbf{T}}
\end{array}\right)=0
$$

with

$$
\mathbf{C}_{\mathbf{P}} \equiv\left(C_{0}, C_{2}, D_{0}, D_{2}\right)^{t}, \quad \mathbf{C}_{\mathbf{T}} \equiv\left(C_{1}, D_{1}\right)^{t},
$$

where the superscript $t$ denotes transposition, and the corresponding matrices are

$$
\mathbf{A}_{\mathbf{P}}=\left(\begin{array}{cccc}
\beta_{4}(q R) & -l(l+1) s^{-2} \beta_{1}(k R) & \widetilde{\beta}_{4}(q R) & -l(l+1) s^{-2} \widetilde{\beta}_{1}(k R) \\
\beta_{1}(q R) & -s^{-2} \beta_{3}(k R) & \widetilde{\beta}_{1}(q R) & -s^{2} \widetilde{\beta}_{3}(k R) \\
\beta_{4}(q a) & -l(l+1) s^{-2} \beta_{1}(k a) & \widetilde{\beta}_{4}(q a) & -l(l+1) s^{-2} \widetilde{\beta}_{1}(k a) \\
\beta_{1}(q a) & -s^{-2} \beta_{3}(k a) & \widetilde{\beta}_{1}(q a) & -s^{-2} \widetilde{\beta}_{3}(k a)
\end{array}\right)
$$




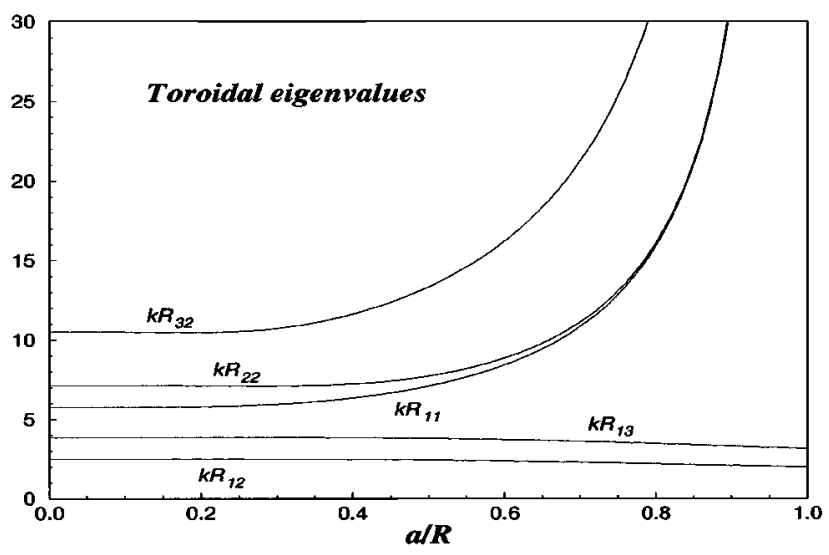

FIG. 1. Functional dependence of the first few toroidal eigenvalues of a hollow sphere on the ratio $a / R$. Solid sphere values $(a=0)$ are found on the intersections with the ordinate axis.

and

$$
\mathbf{A}_{\mathbf{T}}=\left(\begin{array}{ll}
\beta_{1}(k R) & \widetilde{\beta}_{1}(k R) \\
\beta_{1}(k a) & \widetilde{\beta}_{1}(k a)
\end{array}\right) .
$$

Here $s \equiv q / k$, and we have introduced the set of functions

$$
\begin{gathered}
\beta_{0}(z) \equiv j_{l}(z) z^{-2}, \quad \beta_{1}(z) \equiv\left(j_{l}(z) z^{-1}\right)^{\prime}, \quad \beta_{2}(z) \equiv j_{l}^{\prime}(z), \\
\beta_{3}(z) \equiv \frac{1}{2} \beta_{2}(z)-\left\{1-\frac{l(l+1)}{2}\right\} \beta_{0}(z) \\
\beta_{4}(z) \equiv \beta_{2}(z)-\frac{\lambda}{2 \mu} z^{2} \beta_{0}(z),
\end{gathered}
$$

while the tilded ones are their singular counterparts, with $y_{l}$ instead of $j_{l}$ [i.e., $\widetilde{\beta}_{0}(z) \equiv y_{l}(z) z^{-2}$, and so on]. The matrices $\mathbf{A}_{\mathbf{P}}$ and $\mathbf{A}_{\mathbf{T}}$ are functions of $k R$, and depend on the parameter $a / R$, and, in the case of $\mathbf{A}_{\mathbf{P}}$, also on ${ }^{1} s$. The discrete set of $k R$ values that make compatible the system (2.7) constitutes the spectrum of the elastic sphere. We can distinguish two families of normal modes.

(i) Toroidal modes. These are characterized by

$$
\operatorname{det} \mathbf{A}_{\mathbf{T}}=0, \quad \mathbf{C}_{\mathbf{P}}=0 .
$$

Hence they are purely tangential, and their frequencies depend only on the ratio $a / R$. Their amplitudes are

$$
\mathbf{u}_{n l m}^{T}(\mathbf{x})=T_{n l}(r) i \mathbf{L} Y_{l m}(\theta, \phi)
$$

with

\footnotetext{
${ }^{1}$ This parameter is a function of the Poisson ratio $\sigma$; for the usual value $\sigma=1 / 3, s=0.5$ and $\lambda / \mu=2$. These values are assumed, unless otherwise stated.
}

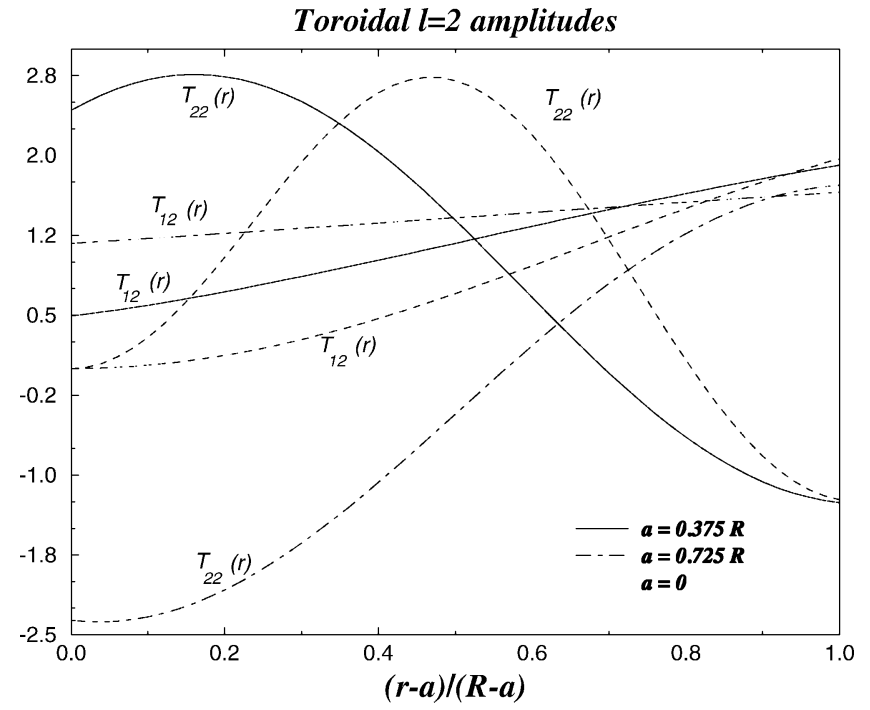

FIG. 2. Toroidal mode radial functions for the first two quadrupole harmonics and a few values of the geometric ratio $a / R$. The magnitude represented in abscissas is such that the region plotted spans radially the material thickness of the hollow sphere.

$$
T_{n l}(r)=C_{1}(n, l)\left\{\widetilde{\beta}_{1}\left(k_{n l} R\right) j_{l}\left(k_{n l} r\right)-\beta_{1}\left(k_{n l} R\right) y_{l}\left(k_{n l} r\right)\right\},
$$

where $C_{1}(n, l)$ is fixed by the chosen normalization. The corresponding eigenvalues are obtained as solutions to the transcendental equation (2.13). For the degenerate limit $a=R$ the equation to be solved is

$$
\operatorname{det}\left(\begin{array}{ll}
\beta_{1}(k R) & \widetilde{\beta_{1}}(k R) \\
\beta_{1}^{\prime}(k R) & \widetilde{\beta}_{1}^{\prime}(k R)
\end{array}\right)=0,
$$

with the prime denoting differentation respect to the argument. Using standard properties of Bessel functions [15], it can be easily shown that

$$
\begin{aligned}
& \beta_{1}(k R) \widetilde{\beta}_{1}^{\prime}(k R)-\widetilde{\beta}_{1}(k R) \beta_{1}^{\prime}(k R) \\
& =(k R)^{-6}\left[(k R)^{2}+2-l(l+1)\right],
\end{aligned}
$$

and, in this case, there is only one eigenvalue for each $l>1$, given by the only root of the above equation, ${ }^{2}$ $\left(k_{l} R\right)^{2}=l(l+1)-2$. Figure 1 displays $k_{n l} R$ as a function of $a / R$ for the first few toroidal modes. The existence of just one mode for each $l>1$ in the thin shell limit shows as a divergence of $k_{n l} R$ when $a / R$ approaches 1 and $n>1$. In Fig. 2 we plot the normalized toroidal amplitudes $T_{n l}(r)$ for two quadrupolar modes and three different values of the parameter $a / R$. We observe that their absolute values at the outer surface show little dependence on the ratio $a / R$.

\footnotetext{
${ }^{2}$ This equation shows explicitly a property shared by all toroidal modes, namely, that their dimensionless eigenvalues $k_{n l} R$ do not depend on the elastic properties of the material.
} 

by

(ii) Spheroidal modes. This second family is characterized

$$
\operatorname{det} \mathbf{A}_{\mathbf{P}}=0, \quad \mathbf{C}_{\mathbf{T}}=0
$$

In this case, the expressions get more involved, as we have to handle a $4 \times 4$ determinant. Once the spectrum $k_{n l}$ is found for a given $a / R$ and $s$, the system (2.7) can be solved for $C_{2} / C_{0}, D_{0} / C_{0}$, and $D_{2} / C_{0}$. If we label these coefficients $p_{0}(n, l), p_{1}(n, l)$, and $p_{2}(n, l)$, the eigenmodes can be written as

$$
\mathbf{u}_{n l m}^{P}=N_{n l}(r) Y_{l m}(\theta, \phi) \mathbf{n}-i E_{n l}(r) \mathbf{n} \times \mathbf{L} Y_{l m}(\theta, \phi),
$$

with

$$
\begin{aligned}
N_{n l}(r)= & C_{0}(n, l)\left[j_{l}^{\prime}\left(q_{n l} r\right)-p_{0}(n, l) \frac{l(l+1)}{q_{n l} r} j_{l}\left(k_{n l} r\right)\right. \\
& \left.+p_{1}(n, l) y_{l}^{\prime}\left(q_{n l} r\right)-p_{2}(n, l) \frac{l(l+1)}{q_{n l} r} y_{l}\left(k_{n l} r\right)\right], \\
E_{n l}(r)= & C_{0}(n, l) \frac{1}{q_{n l} r}\left[j_{l}\left(q_{n l} r\right)-p_{0}(n, l)\left\{k_{n l} r j_{l}\left(k_{n l} r\right)\right\}^{\prime}\right. \\
& \left.+p_{1}(n, l) y_{l}\left(q_{n l} r\right)-p_{2}(n, l)\left\{k_{n l} r y_{l}\left(k_{n l} r\right)\right\}^{\prime}\right],
\end{aligned}
$$

where $C_{0}(n, l)$ is, again, free up to normalization. The spectrum for the degenerate case $a=R$ is given by the solutions to which happens to have two solutions for each value of $l$ when $l>1$ and only one $\operatorname{root}^{3}$ for $l<2$.

Plotting $k_{n l} R$ as a function of $a / R$, we see that the third and higher roots diverge as the inner radius approaches $R$; see Figs. 3 and 4. Figures 5-7 show the normalized radial functions for a few spheroidal modes and values of $a / R$. As in the toroidal case, their values at $r=R$ (where measurements using transducers are to be made eventually) are nearly independent of $a / R$.

\section{CROSS SECTION FOR THE HOLLOW SPHERE}

A convenient way to characterize a resonant detector sensitivity is through its GW energy absorption cross section, defined as

$$
\sigma_{\mathrm{abs}}(\omega)=\frac{\Delta E_{a}(\omega)}{\Phi(\omega)},
$$

where $\Delta E_{a}(\omega)$ is the energy absorbed by the detector at frequency $\omega$, and $\Phi(\omega)$ is the incident flux density expressed, e.g., in $\mathrm{W} / \mathrm{m}^{2} \mathrm{~Hz}$. Estimation of $\sigma_{\mathrm{abs}}(\omega)$ requires a hypothesis about the underlying gravitation theory to calculate $\Phi(\omega)$ and specification of the antenna's geometry to

${ }^{3}$ The purely radial case $l=0$ is simpler, because the eigenvalue equation (2.21) becomes

$$
\beta_{4}(q R) \widetilde{\beta_{4}^{\prime}}(q R)-\beta_{4}^{\prime}(q R) \widetilde{\beta_{4}}(q R)=0,
$$

and has only one solution, namely, $q R=(\mu / \lambda) \sqrt{3-\mu / \lambda}$. Unlike toroidal eigenvalues, spheroidal ones do depend on $\mu / \lambda$. calculate $\Delta E_{a}(\omega)$. Here we shall assume that general relativity is the correct gravitation theory and proceed to calculate the oscillation energy of the solid as a consequence of its excitation by an incoming GW, which we shall naturally identify with $\Delta E_{a}(\omega)$. We briefly sketch the details of the process now.

As shown in [8], an elastic solid's response to a GW force can be expressed by a very general formula, which is easily particularized to a spherically symmetric body such as the solid sphere or the hollow sphere. In both cases, as we have

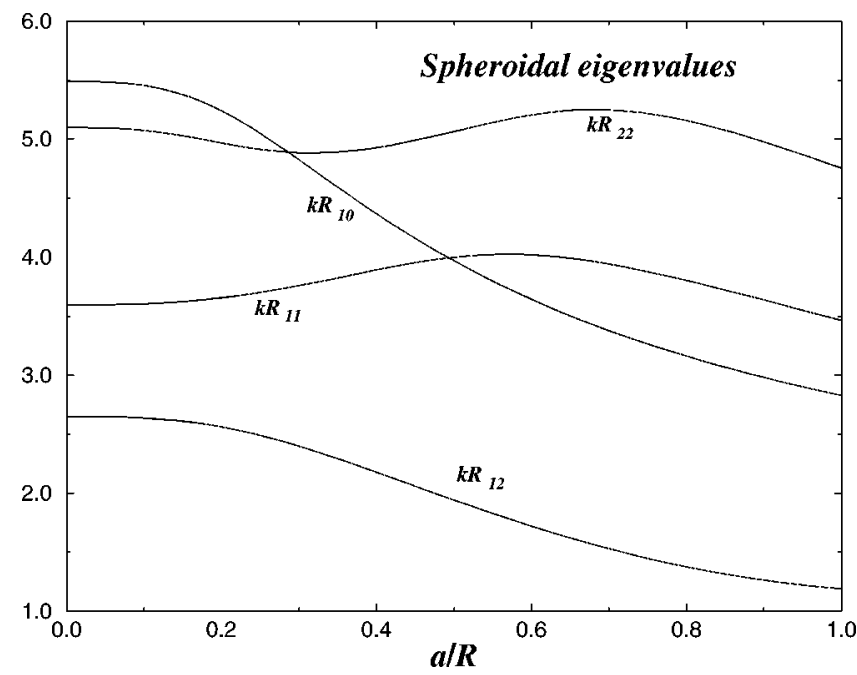

FIG. 3. Functional dependence of the first few spheroidal eigenvalues of a hollow sphere on the ratio $a / R$. Solid sphere values $(a=0)$ are found on the intersections with the ordinate axis. 


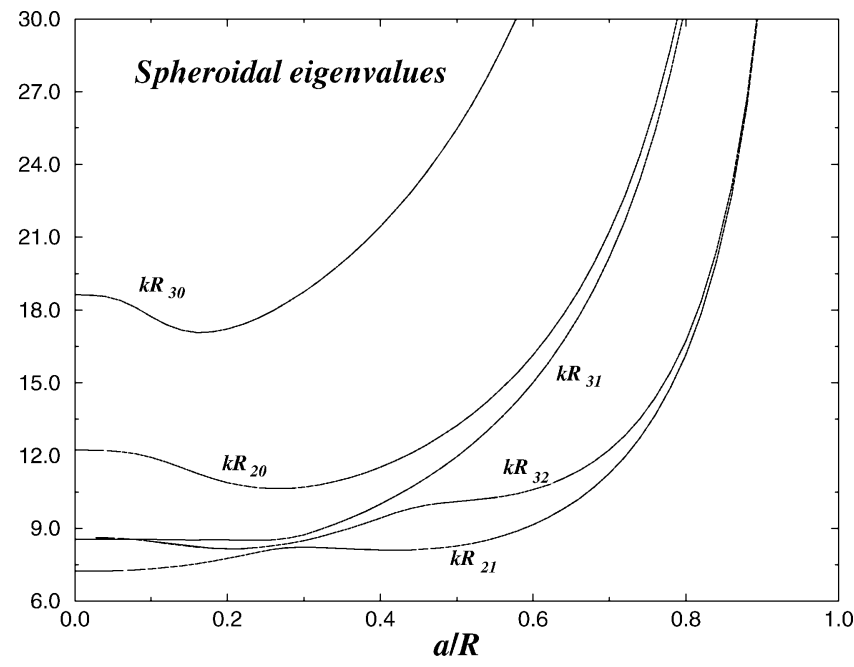

FIG. 4. Functional dependence of higher spheroidal eigenvalues of a hollow sphere on the ratio $a / R$. The harmonics in this graph do not exist in the thin shell limit, and this shows as divergences as $a$ approaches $R$.

just seen, the vibration eigenmodes belong to two families (spheroidal and toroidal), but GW's only couple to quadrupole spheroidal harmonics. If the frequencies of these modes are noted by $\omega_{n 2}(n=1$ for the lowest value, $n=2$ for the next, etc.) and the corresponding wave functions by $\mathbf{u}_{n 2 m}(\mathbf{x})$, then the elastic displacements are given by

$$
\mathbf{u}(\mathbf{x}, t)=\sum_{n=1}^{\infty} \frac{b_{n}}{\omega_{n 2}}\left[\sum_{m=-2}^{2} \mathbf{u}_{n 2 m}(\mathbf{x}) g_{n 2}^{(m)}(t)\right],
$$

where

$$
g_{n l}^{(m)}(t) \equiv \int_{0}^{t} g^{(m)}\left(t^{\prime}\right) \sin \omega_{n 2}\left(t-t^{\prime}\right) d t^{\prime} \quad(m=-2, \ldots, 2)
$$

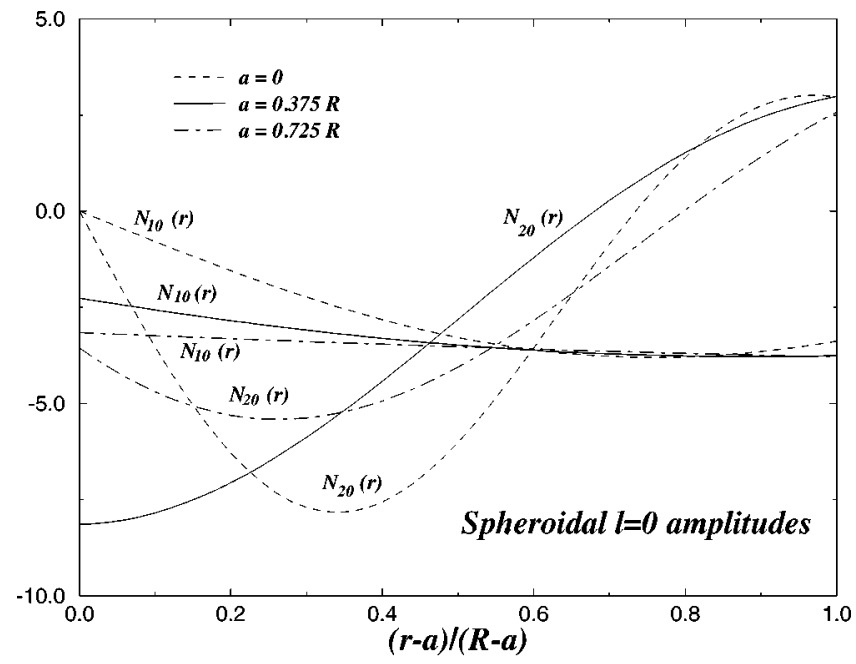

FIG. 5. Spheroidal mode radial $N$-functions — see Eq. (2.19) for the first two monopole harmonics and a few values of the geometric ratio $a / R$. The magnitude represented in abscissas is such that the region plotted spans radially the material thickness of the hollow sphere.

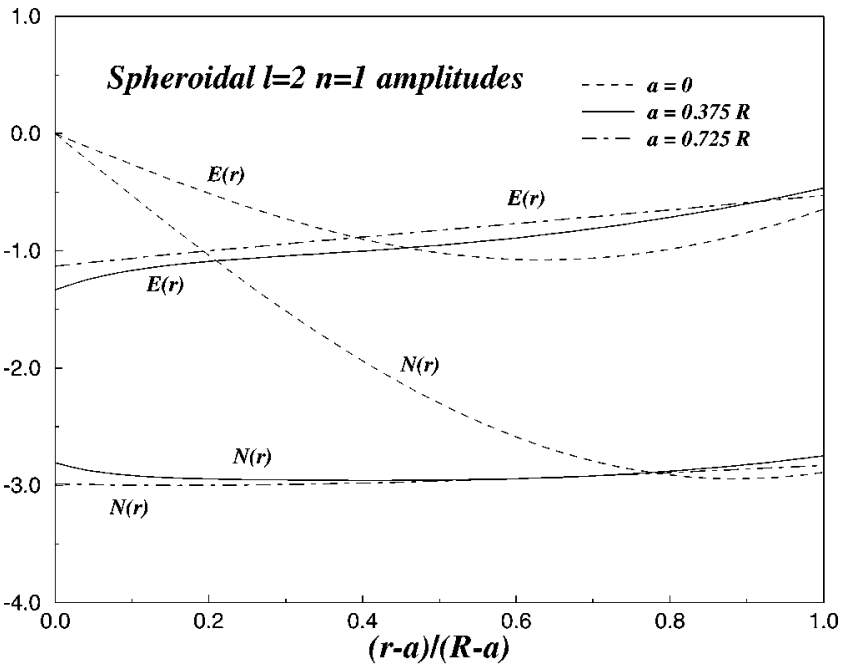

FIG. 6. Spheroidal mode radial functions-see Eqs. (2.19) and (2.20) — for the first quadrupole harmonic.

and $g^{(m)}(t)$ are the quadrupole components of the Riemann tensor, while $b_{n}$ is an overlapping integral factor of the GW's tidal coefficient over the solid's extension. Much like in the case of a solid sphere, it has dimensions of length and is given by a definite integral of the radial terms in the wave function $\mathbf{u}_{n 2 m}(\mathbf{x})$; more specifically,

$$
\begin{aligned}
\frac{b_{n}}{R} & =-\frac{\rho}{M} \int_{a}^{R} r^{3}\left[N_{n 2}(r)+3 E_{n 2}(r)\right] d r \\
& =-\frac{C_{0}(n, 2)}{4 \pi q_{n 2} R}\left[G_{2}(R)-G_{2}(a)\right],
\end{aligned}
$$

where we have introduced the dimensionless function

$$
\begin{aligned}
G_{2}(z) \equiv & \frac{z^{3}}{R^{3}-a^{3}}\left[j_{2}\left(q_{n 2} z\right)+p_{1}(n, 2) y_{2}\left(q_{n 2} z\right)\right. \\
& \left.-3 p_{0}(n, 2) j_{2}\left(k_{n 2} z\right)-3 p_{2}(n, 2) y_{2}\left(k_{n 2} z\right)\right]
\end{aligned}
$$

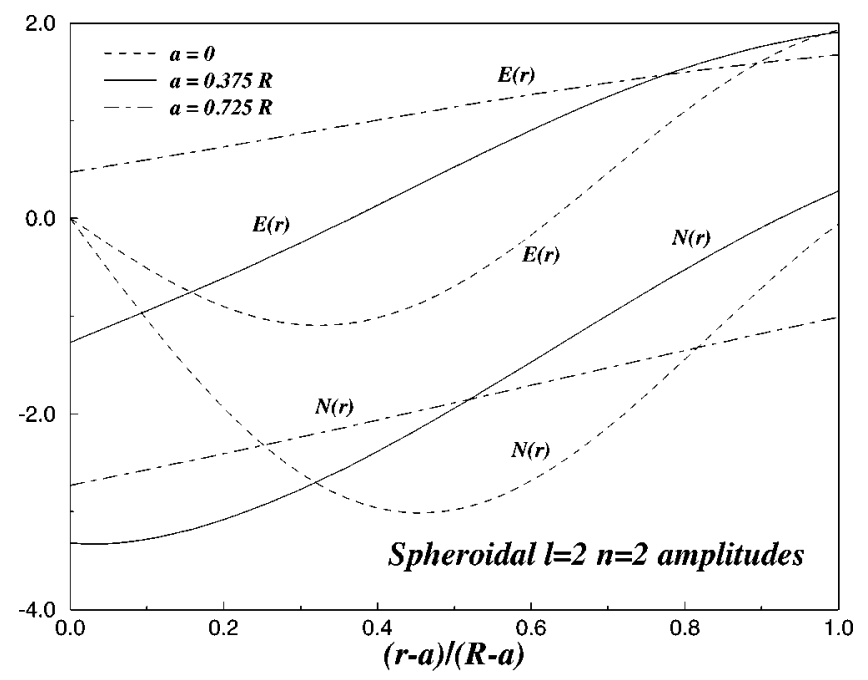

FIG. 7. Spheroidal mode radial functions-see Eqs. (2.19) and (2.20) — for the second quadrupole harmonic. 
and assumed the following normalization for the wave functions:

$$
\begin{aligned}
\int_{\text {solid }}\left|\mathbf{u}_{n l m}\right|^{2} \rho d^{3} \mathbf{x} & =\int_{a}^{R} r^{2} d r \rho\left[N_{l n}^{2}(r)+l(l+1) E_{l n}^{2}(r)\right] \\
& =M
\end{aligned}
$$

The calculation of $\Delta E_{a}(\omega)$ can now be pursued along the lines set up in Ref. [8]: The Fourier transform $\mathbf{U}(\mathbf{x}, \omega)$ of the response function $\mathbf{u}(\mathbf{x}, t)$ of Eq. (3.2) is calculated, whereby the spectral energy density can be obtained as

$$
W(\omega)=\frac{1}{T} \int_{\text {solid }} \frac{1}{2} \omega^{2}|\mathbf{U}(\mathbf{x}, \omega)|^{2} \rho d^{3} x,
$$

where $T$ is the integration time of the signal in the detector. The energy deposited by the GW in the $n$th quadrupole mode is hence calculated by integration of this spectral density over the linewidth of the mode. It is readily found that

$$
\Delta E_{a}\left(\omega_{n 2}\right)=\frac{1}{2} M b_{n}^{2} \sum_{m=-2}^{2}\left|G^{(m)}\left(\omega_{n 2}\right)\right|^{2},
$$

where $G^{(m)}(\omega)$ is the Fourier transform of $g^{(m)}(t)$.

The GW flux in the denominator of Eq. (3.1) is (clearly) proportional to the sum in the right-hand side (RHS) of Eq. (3.8), the proportionality factor being in turn proportional to $\omega^{2}$ —see [8] for a detailed discussion—so we finally obtain

$$
\sigma_{n} \equiv \sigma_{\mathrm{abs}}\left(\omega_{n 2}\right)=\frac{16 \pi^{2}}{15} \frac{G M v_{t}^{2}}{c^{3}}\left(k_{n 2} b_{n}\right)^{2},
$$

where $v_{t}^{2}=\mu / \rho, M$ is the detector's mass and $G$ is Newton's constant. This equation allows relatively easy numerical evaluation of the cross sections, as well-defined computer programs can be written for the purpose.

As we have seen in Sec. II above, the eigenvalues and wave functions of a hollow sphere only depend on the ratio $a / R$, and therefore so does the quantity $\left(k_{n 2} b_{n}\right)$ in Eq. (3.9). So the cross section $\sigma_{n}$ only depends on that ratio, too, once a suitable unit of mass is adopted for reference. In Figs. 8 and 9 we plot $\sigma_{n}$ for the first two quadrupole modes of the hollow sphere in two different circumstances: In Fig. 8 we assume a hollow sphere of fixed outer radius-thus its mass decreases with thickness - and in Fig. 9 we have instead assumed that the mass of the hollow sphere is fixed, so that its geometrical size increases as it gets thinner. In either case we see that, for the higher mode, the maximum cross section does not happen at $a=0$, but at some intermediate inner radius: For $a \approx 0.37745 R$, the cross section for the second quadrupole mode equals that of the first, and we have the possibility of working with a detector with the same (high) sensitivity at two frequencies.

\section{SENSITIVITY TO GW SIGNALS}

We assume that the mechanical oscillations induced in a resonant mass by the interaction with the GW are transformed into electrical signals by a set of identical noiseless transducers (for the sake of simplicity, we consider here non-

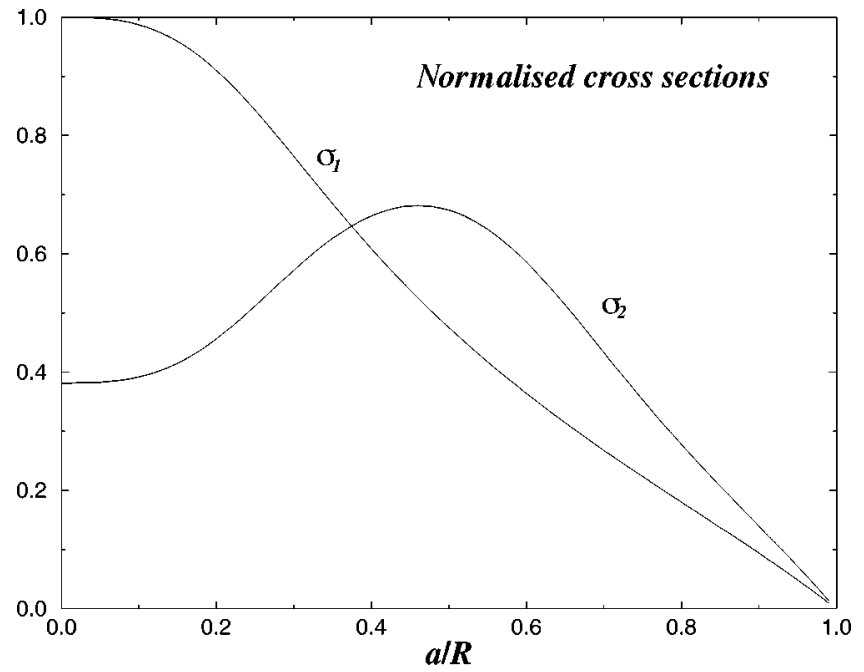

FIG. 8. Cross sections of a hollow sphere in its first two quadrupole modes as a function of thickness. Values are referred to the cross section of a solid sphere in its first quadrupole resonance, whose radius is assumed to be equal to the outer radius of the hollow sphere.

resonant transducers), perfectly matched to electronic amplifiers with noise temperature $T_{n}$. Unavoidably, Brownian motion noise associated with dissipation in the antenna and electronic noise from the amplifiers limit the sensitivity of the detector. We refer the reader to [16-18] for a complete discussion on the sensitivity of resonant-mass detectors and report here only a few basic formulas for the evaluation of the detector sensitivity to various signals.

The total noise at the output of each resonant mode can be seen as due to an input noise generator having spectral density of strain $S_{h}(f)$, acting on a noiseless oscillator. $S_{h}(f)$ represents the input GW spectrum that would produce a signal equal to the noise spectrum actually observed at the output of the detector instrumentation. In a resonant-mass detector, this function is a resonant curve and can be characterized by its value at resonance $S_{h}\left(f_{n}\right)$ and by its half height width. $S_{h}\left(f_{n}\right)$ can be written as

$$
S_{h}\left(f_{n}\right)=\frac{G}{c^{3}} \frac{4 k T_{e}}{\sigma_{n} Q_{n} f_{n}} .
$$

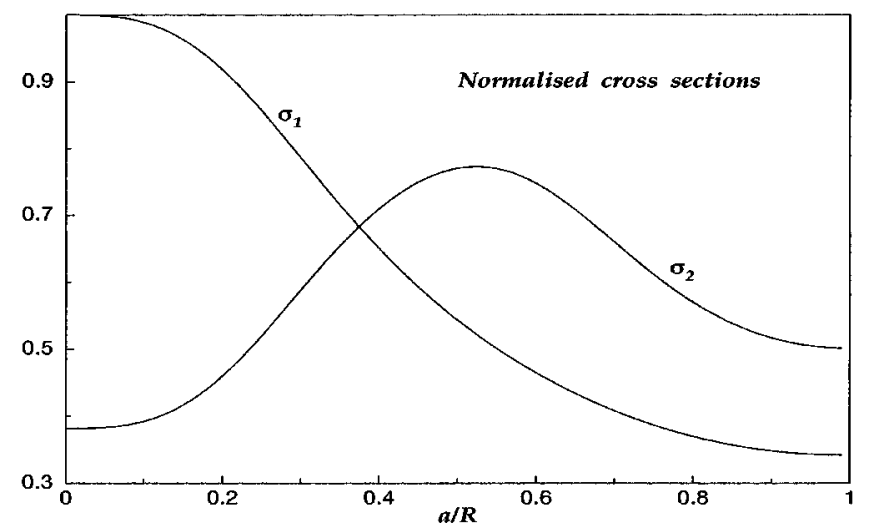

FIG. 9. Cross sections of a hollow sphere in its first two quadrupole modes as a function of thickness. Values are referred to the cross section of a solid sphere in its first quadrupole resonance, whose mass equals that of the hollow sphere. 
TABLE I. Main features and sensitivities for several hypothetical hollow spheres of two different materials.

\begin{tabular}{cccccccccc}
\hline \hline & $M$ (ton) & $2 R(\mathrm{~m})$ & $(R-a)(\mathrm{cm})$ & $f_{1}(\mathrm{~Hz})$ & $f_{2}(\mathrm{~Hz})$ & $\sigma_{1}\left(\mathrm{~m}^{2} \mathrm{~Hz}\right)$ & $\sigma_{2}\left(\mathrm{~m}^{2} \mathrm{~Hz}\right)$ & $\sqrt{S_{h 1}}\left(\mathrm{~Hz}^{-1 / 2}\right)$ & $\left.\sqrt{S_{h 2}}(\mathrm{~Hz})^{-1 / 2}\right)$ \\
\hline CuAl & 200 & 4 & 81 & 395 & 1188 & $1.5 \times 10^{-23}$ & $2.4 \times 10^{-23}$ & $4.7 \times 10^{-24}$ & $3.7 \times 10^{-24}$ \\
& 200 & 6 & 25 & 191 & 753 & $1.1 \times 10^{-23}$ & $1.7 \times 10^{-23}$ & $5.4 \times 10^{-24}$ & $4.4 \times 10^{-24}$ \\
& 100 & 4 & 31 & 302 & 1161 & $5.8 \times 10^{-24}$ & $8.8 \times 10^{-24}$ & $7.5 \times 10^{-24}$ & $6.1 \times 10^{-24}$ \\
& 100 & 6 & 12 & 185 & 738 & $5.6 \times 10^{-24}$ & $8.2 \times 10^{-24}$ & $7.7 \times 10^{-24}$ & $6.3 \times 10^{-24}$ \\
& 40 & 3 & 22 & 399 & 1543 & $2.3 \times 10^{-24}$ & $3.5 \times 10^{-24}$ & $1.2 \times 10^{-23}$ & $9.7 \times 10^{-24}$ \\
& 40 & 4 & 11 & 281 & 1115 & $2.2 \times 10^{-24}$ & $3.3 \times 10^{-24}$ & $1.2 \times 10^{-23}$ & $1.0 \times 10^{-23}$ \\
Al 5056 & 200 & 6 & 90 & 273 & 935 & $1.8 \times 10^{-23}$ & $2.9 \times 10^{-23}$ & $4.3 \times 10^{-24}$ & $3.4 \times 10^{-24}$ \\
& 100 & 6 & 37 & 230 & 896 & $7.7 \times 10^{-24}$ & $1.2 \times 10^{-23}$ & $6.6 \times 10^{-24}$ & $5.4 \times 10^{-24}$ \\
& 40 & 4 & 35 & 361 & 1370 & $3.2 \times 10^{-24}$ & $4.8 \times 10^{-24}$ & $1.0 \times 10^{-23}$ & $8.3 \times 10^{-24}$ \\
& 40 & 6 & 14 & 218 & 866 & $3.0 \times 10^{-24}$ & $4.4 \times 10^{-24}$ & $1.0 \times 10^{-23}$ & $8.7 \times 10^{-24}$ \\
\hline \hline
\end{tabular}

Here $T_{e}$ is the thermodynamic temperature of the detector plus a back-action contribution from the amplifiers, and $Q_{n}$ is the quality factor of the mode.

The half height width of $S_{h}(f)$ gives the bandwidth of the resonant mode:

$$
\Delta f_{n}=\frac{f_{n}}{Q_{n}} \Gamma_{n}^{-1 / 2} .
$$

Here, $\Gamma_{n}$ is the ratio of the wideband noise in the $n$th resonance bandwidth to the narrowband noise,

$$
\Gamma_{n} \simeq \frac{T_{n}}{2 \beta_{n} Q_{n} T_{e}}
$$

where $\beta_{n}$ is the transducer coupling factor, defined as the fraction of the total mode energy available at the transducer output.

In practice $\Gamma_{n} \ll 1$ and the bandwidth is much larger than the pure resonance linewidth $f_{n} / Q_{n}$. In the limit $\Gamma_{n} \rightarrow 0$, the bandwidth becomes infinite. The bandwidth of the present resonant bars is of the order of a few $\mathrm{Hz}$ [1]. If a quantumlimited readout system were available, values of the order of $100 \mathrm{~Hz}$ could be reached $[19,20]$.

Equations (4.1) and (4.2) can be used to characterize the sensitivity of the quadrupole modes of a hollow spherical resonant-mass detector. The optimum performance is obtained by filtering the output with a filter matched to the signal. The energy signal-to-noise ratio (SNR) of the filter output is given by the well-known formula

$$
\mathrm{SNR}=\int_{-\infty}^{+\infty} \frac{|H(f)|^{2}}{S_{h}(f)} d f
$$

where $H(f)$ is the Fourier transform of $h(t)$.

We now report the SNR of a hollow spherical detector for various GW signals. To be specific, we shall assume that the thermodynamic temperature of the detector can be reduced to below $50 \mathrm{mK}$ and that the quality factors of the modes are of the order of $10^{7}$, so that the overall detector noise will be dominated by the electronic amplifier noise. If we express the energy of the latter as a multiple of the quantum limit, i.e., $k T_{n}=N \hbar \omega$, then the strain spectral density becomes

$$
S_{h}\left(f_{n}\right) \simeq \frac{G}{c^{3}} \frac{4 \pi \beta_{n} N \hbar}{\sigma_{n}} .
$$

In these conditions the fractional bandwidth $\Delta f_{n} / f_{n}$ becomes of the order of $\beta_{n}$ that we assume of about 0.1 . We shall consider hollow spheres made of the usual aluminum alloy Al 5056 and of a recently investigated copper alloy $(\mathrm{CuAl})$ [21]. Table I displays numerical values of the most relevant parameters for a few example detectors with a noise level equal to the quantum limit, i.e., $N=1$.

\section{A. Bursts}

We model the burst signal as a featureless waveform, rising quickly to an amplitude $h_{0}$ and lasting for a time $\tau_{g}$ much shorter than the detector integration time $\Delta t=\Delta f_{n}^{-1}$. Its Fourier transform will be considered constant within the detector bandwidth: $H(f) \simeq H\left(f_{n}\right)=H_{0}$. From Eq. (4.4) we get

$$
\mathrm{SNR}=\frac{2 \pi \Delta f_{n} H_{0}^{2}}{S_{h}\left(f_{n}\right)} .
$$

For SNR $=1$ and using the equation $H_{0}^{\min }=h_{0}^{\min } \tau_{g}$, we find

$$
\left(h_{0}^{\min }\right)_{\text {burst }}=\tau_{g}^{-1}\left[\frac{S_{h}\left(f_{n}\right)}{2 \pi \Delta f_{n}}\right]^{1 / 2} .
$$

The level $h_{0}^{\min } \simeq 10^{-22}$ can be reached by the lowest-order mode of a typical large hollow spherical detector such as the one being considered. The GW luminosity of burst sources is still largely unknown, and so it is difficult to accurately estimate their detectability. The above sensitivity is however likely to enable the detection of GW collapses in the Virgo cluster for an energy conversion of $10^{-4} M_{\odot}$ into a millisecond GW burst. See Table II for a few specific examples.

\section{B. Monochromatic signals}

We consider a sinusoidal wave of amplitude $h_{0}$ and frequency $f_{s}$ constant over the observation time $t_{m}$. The Fourier transform amplitude at $f_{n}$ is $1 / 2 h_{0} t_{m}$ with a bandwidth given by $t_{m}^{-1}$. The SNR can be written as 
TABLE II. Sensitivity to burst and monochromatic (integrated for $1 \mathrm{y}$ ) GW signals of a few hollow spheres of two different materials.

\begin{tabular}{lccccccccc}
\hline \hline & $M($ ton $)$ & $2 R(\mathrm{~m})$ & $(R-a)(\mathrm{cm})$ & $f_{1}(\mathrm{~Hz})$ & $f_{2}(\mathrm{~Hz})$ & $\left(h_{01}^{\min }\right)_{\text {burst }}$ & $\left(h_{02}^{\min }\right)_{\text {burst }}$ & $\left(h_{01}^{\min }\right)_{m}$ & $\left(h_{02}^{\min }\right)_{m}$ \\
\hline CuAl & 200 & 6 & 25 & 191 & 753 & $4.9 \times 10^{-22}$ & $2.0 \times 10^{-22}$ & $1.4 \times 10^{-27}$ & $1.1 \times 10^{-27}$ \\
& 100 & 4 & 31 & 302 & 1161 & $5.5 \times 10^{-22}$ & $2.3 \times 10^{-22}$ & $1.9 \times 10^{-27}$ & $1.5 \times 10^{-27}$ \\
Al 5056 & 100 & 6 & 37 & 230 & 896 & $5.5 \times 10^{-22}$ & $2.3 \times 10^{-22}$ & $1.7 \times 10^{-27}$ & $1.4 \times 10^{-27}$ \\
\hline \hline
\end{tabular}

$$
\mathrm{SNR}=\frac{t_{m} h_{0}^{2}}{2 S_{h}\left(f_{n}\right)}\left\{1+\Gamma_{n}\left[Q^{2}\left(1-f_{s}^{2} / f_{n}^{2}\right)+f_{s}^{2} / f_{n}^{2}\right]\right\}
$$

For SNR $=1$ we obtain a minimum detectable value of $h_{0}$, which at $f_{s}=f_{n}$ is

$$
\left(h_{0}^{\min }\right)_{m}=\left[\frac{2 S_{h}\left(f_{n}\right)}{t_{m}}\right]^{1 / 2}
$$

See Table II for a few specific examples. For instance, the nearby pulsar [22] PSR J0437-4715, at a distance of $150 \mathrm{pc}$, might emit at $347 \mathrm{~Hz}$ a GW amplitude (optimistically) of $2 \times 10^{-26}$. This would give SNR $=100$ on a hollow spherical detector having $M=100$ tons after integrating the signal for $1 \mathrm{y}$.

\section{Chirps}

We consider here the interaction of the hollow spherical detector with the waveform emitted by a binary system, consisting of either neutron stars or black holes, in the inspiral phase. The system, in the Newtonian regime, has a clean analytic behavior, and emits a waveform of increasing amplitude and frequency that can sweep up to the $\mathrm{kHz}$ range of frequency.

From the resonant-mass detector viewpoint, the chirp signal can be treated as a transient GW, depositing energy in a time-scale short with respect to the detector damping time [23]. We can then use Eq. (4.6) to evaluate the SNR, where the Fourier transform $H\left(f_{n}\right)$ at the resonant frequency $f_{n}$ can be explicitly written as

$$
\begin{aligned}
H\left(f_{n}\right)= & \left\{\left[\int h(t) \cos \left(2 \pi f_{n} t\right) d t\right]^{2}\right. \\
& \left.+\left[\int h(t) \sin \left(2 \pi f_{n} t\right) d t\right]^{2}\right\}^{1 / 2},
\end{aligned}
$$

with $h(t)$ indicating $h_{+}(t)$ or $h_{\times}(t)$. Substituting into Eq. (4.10) the well-known chirp waveforms for an optimally oriented orbit of zero eccentricity in the Newtonian approximation [18], the SNR for chirp detection is [24]

$$
\mathrm{SNR}=\frac{2^{1 / 3} 5}{12} \frac{G^{5 / 3}}{c^{3}} \frac{\pi^{2} \Delta f_{n}}{S_{h}\left(f_{n}\right)} \frac{1}{r^{2}} M_{c}^{5 / 3}\left(2 \pi f_{n}\right)^{-7 / 3}
$$

$M_{c}$ is the chirp mass defined as $M_{c}=\left(m_{1} m_{2}\right)^{3 / 5}\left(m_{1}\right.$ $\left.+m_{2}\right)^{-1 / 5}$, where $m_{1}$ and $m_{2}$ are the masses of the two compact objects and $r$ is the distance to the source. The chirp mass is the only parameter that determines the frequency sweep rate of the chirp signal in the Newtonian approximation and can be determined by a double passage technique
[24]: Much like in a solid sphere detector, one can measure the time delay $\tau_{2}-\tau_{1}$ between excitations of the first and second quadrupole modes on a hollow spherical detector to calculate the chirp mass through equation

$$
M_{c}=2^{8 / 5}\left(\frac{5}{256}\right)^{3 / 5} \frac{c^{3}}{G}\left(\frac{\omega_{2}^{-8 / 3}-\omega_{1}^{-8 / 3}}{\tau_{2}-\tau_{1}}\right)^{3 / 5},
$$

where $\omega_{1}$ and $\omega_{2}$ are the angular frequencies of the first and second quadrupole modes, respectively. Time delays are of the order of a fraction of a second for the hollow spheres considered in this paper, well within the timing possibilities of resonant mass detectors [25].

Another consequence of the multimode and multifrequency nature of a spherically symmetric detector is the possibility to determine the orbit orientation by the measurement of the relative proportion of the two polarization amplitudes, and thereby the distance to the source and the intrinsic GW amplitudes [24]. See Figs. 10 and 11 for a specific example referring to optimally oriented circular orbits.

Because of the Newtonian approximation, Eqs. (4.11) and (4.12) become inaccurate near coalescence. In analogy with previous analyses $[23,24]$, we limit our considerations to the frequency at which there are still five cycles remaining in the waveform until coalescence. The highest chirp mass values

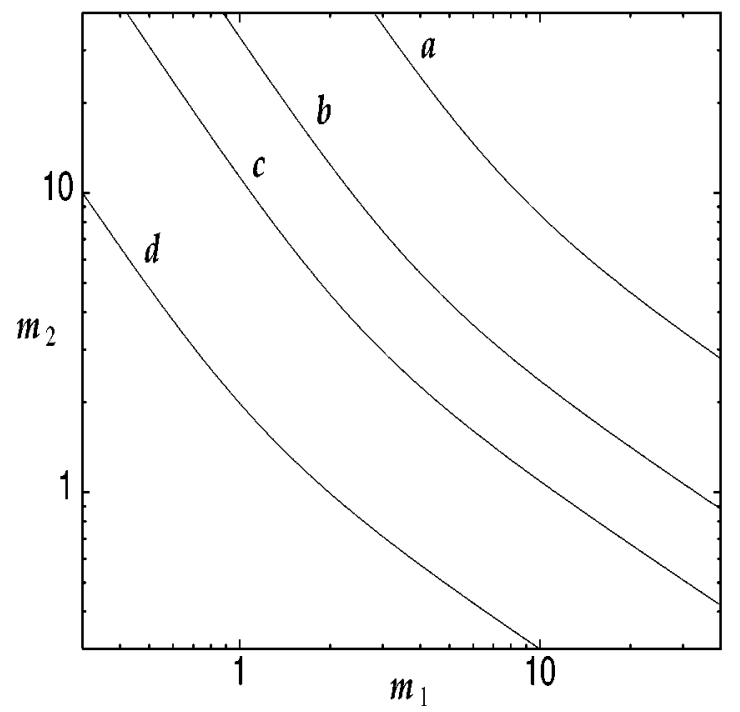

FIG. 10. Contours of constant chirp mass $M_{c}$ in $m_{1}, m_{2}$ space. At each chirp mass corresponds the maximum distance $r$ at which the chirp can be observed with a $\mathrm{SNR}=10$ by a 200-ton $\mathrm{CuAl}$ hollow sphere, $6 \mathrm{~m}$ in diameter, at its first resonance frequency $f_{1}=191 \mathrm{~Hz}$. The reported chirp mass values (in units of solar masses) and the corresponding maximum distances are $M_{c}=8.0$, $r=214 \mathrm{Mpc}$ (curve $a), M_{c}=4.0, r=119 \mathrm{Mpc}$ (curve $\left.b\right), M_{c}=2.6$, $r=84 \mathrm{Mpc}($ curve $c)$, and $M_{c}=1.2, r=45 \mathrm{Mpc}($ curve $d)$. 


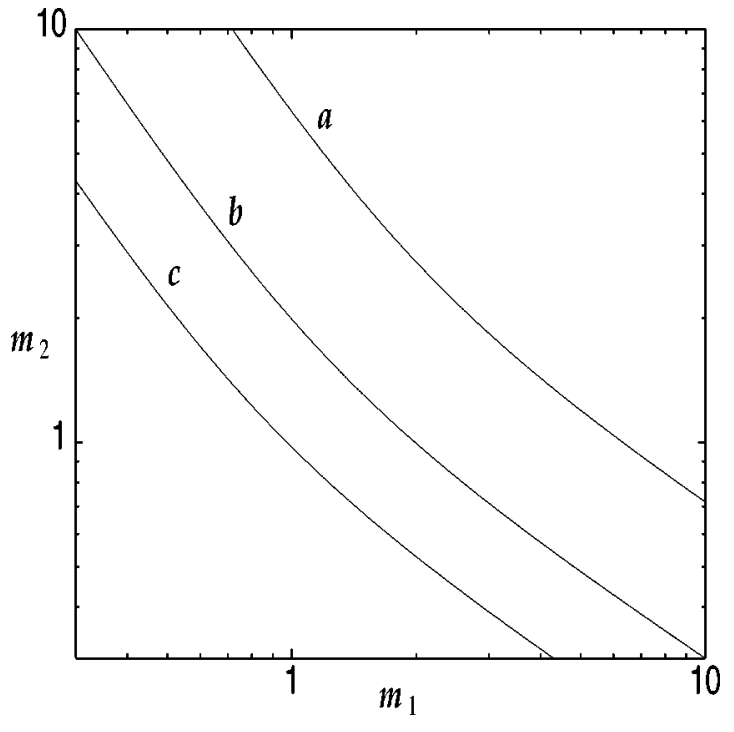

FIG. 11. Contours of constant $M_{c}$ for the same hollow sphere as in Fig. 10, observing the chirp at the second resonance frequency $f_{2}=753 \mathrm{~Hz}$, with $\mathrm{SNR}=10$. The chirp masses and the maximum distances are $M_{c}=2.0, r=131 \mathrm{Mpc}$ (curve $a$ ), $M_{c}=1.2, r=86 \mathrm{Mpc}$ (curve $b$ ), and $M_{c}=0.9, r=65 \mathrm{Mpc}$ (curve $c$ ). If the doublepassage technique is applied, the delay times between the excitation of the first and the second mode by the chirps of the given mass are $160 \mathrm{~ms}$ (curve $a$ ), $373 \mathrm{~ms}$ (curve $b$ ), and $648 \mathrm{~ms}$ (curve $c$ ).

reported in the figures are determined by the requirement that the five-cycle frequency of the source be larger than the resonant frequencies of the detector.

\section{Stochastic background}

In this case $h(t)$ is a random function and we assume that its power spectrum, indicated by $S_{\mathrm{GW}}(f)$, is flat and its energy density per unit logarithmic frequency is a fraction $\Omega_{\mathrm{GW}}(f)$ of the closure density $\rho_{c}$ of the Universe:

$$
\frac{d \rho_{\mathrm{GW}}}{d \ln f}=\Omega_{\mathrm{GW}} \rho_{c} .
$$

$S_{\mathrm{GW}}(f)$ is given by

$$
S_{\mathrm{GW}}(f)=\frac{2 G}{\pi} f^{-3} \Omega_{\mathrm{GW}}(f) \rho_{c} .
$$

The measured noise spectrum $S_{h}(f)$ of a single resonantmass detector automatically gives an upper limit to $S_{\mathrm{GW}}(f)$ [and hence to $\Omega_{\mathrm{GW}}(f)$ ].

Two different detectors with overlapping bandwidth $\Delta f$ will respond to the background in a correlated way. The SNR of a GW background in a cross correlation experiment between two detectors located near one another and having a power spectral density of noise $S_{h}^{1}(f)$ and $S_{h}^{2}(f)$ is [26]

$$
\mathrm{SNR}=\left(\frac{S_{\mathrm{GW}}^{2}}{S_{h}^{1} S_{h}^{2}} \Delta f t_{m}\right)^{1 / 4}
$$

where $t_{m}$ is the total measuring time.
Detectors located some distance apart do not correlate quite so well because GW's coming from within a certain cone about the line joining the detectors will reach one of them before the other. The falloff in the correlation with separation is a function of the ratio of the wavelength to the separation and has been studied for pairs of bars, pairs of interferometers [27,28], and pair of spherical detectors [29].

Assuming two identical large hollow spherical detectors are colocated for optimum correlation, the background will reach a $\mathrm{SNR}=1$ if $\Omega_{\mathrm{GW}}$ is

$$
\begin{aligned}
\Omega_{\mathrm{GW}} \simeq & 10^{-9} \times\left(\frac{f_{n}}{200 \mathrm{~Hz}}\right)^{3}\left(\frac{\sqrt{S_{h}^{1}\left(f_{n}\right)}}{10^{-24} \mathrm{~Hz}^{-1 / 2}}\right) \\
& \times\left(\frac{\sqrt{S_{h}^{2}\left(f_{n}\right)}}{10^{-24} \mathrm{~Hz}^{-1 / 2}}\right)\left(\frac{20 \mathrm{~Hz}}{\Delta f_{n}}\right)^{1 / 2}\left(\frac{10^{7} \mathrm{sec}}{t_{m}}\right)^{1 / 2}
\end{aligned}
$$

where the Hubble constant has been assumed $100 \mathrm{~km} \mathrm{~s}^{-1}$.

Hollow spherical detectors can set very interesting limits on the GW background. In particular, following recent estimations based on cosmological string models [30], it emerges that experimental measurements performed at the level of sensitivity attainable with these detectors would be true tests of Planck-scale physics.

Equation (4.15) and (4.16) hold for whichever crosscorrelation experiment between two GW detectors adjacent and aligned for optimum correlation. An interesting consequence is that the sensitivity of a hollow sphereinterferometer observatory will be unprecedented. It can be worthwhile to build a hollow spherical mass detector close to a large interferometer, like LIGO or VIRGO, to perform stochastic searches [31].

\section{CONCLUSIONS}

In this paper we have been mainly concerned with the problem of how an elastic hollow sphere responds to a GW signal impinging on it. To address this problem we have developed an analytical procedure to fully sort out the eigenfrequencies and eigenmodes of that kind of solid, and then applied it to calculate the GW absorption cross section for arbitrary thicknesses and materials of our solid.

When realistic hypotheses are made regarding the size and material of a possible GW detector of this shape, we have seen that a hollow sphere can be advantageous in several respects. It has all the features associated with its symmetry, such as omnidirectionality, and the capability to determine the source direction and wave polarization. Also, its quadrupole frequencies are below those of an equally massive solid sphere, thus making the low-frequency range accessible to this antenna with good sensitivity. We have investigated the system response to the classical GW signal sources (bursts, chirps, continuous, and stochastic) for several sizes and materials, and seen that interesting signal-tonoise ratios are attainable with such a detector. Also, its bandwidth partly overlaps with that of the projected large interferometers [32,33], and so potentially both kinds of de- 
tectors can be operated simultaneously to make hybrid GW observatories of unprecedented sensitivity and signal characterization power.

While it seems possible to cool a 100-ton solid sphere down to $50 \mathrm{mK}$ [34], the possibility of cooling a large hollow sphere at such low temperatures, as well as the fabrication technique and the influence of cosmic rays on a lowtemperature GW detector of that shape and dimensions, is currently under investigation.

\section{ACKNOWLEDGMENTS}

We are grateful to S. Merkowitz and S. Vitale for helpful discussions, and also to A. Semeonov from the Kapitza Institute for Physical Problems, Moscow. One of us (G.F.) has received financial support from the Dutch NWO, two of us (J.A.L. and J.A.O.) from the Spanish Ministry of Education through Contract No. PB93-1050, and two of us (E.C. and V.F.) from the Istituto Nazionale di Fisica Nucleare.
[1] Gravitational Wave Experiments, Proceedings of the First Edoardo Amaldi Conference, Frascati, 1994, edited by E. Coccia, G. Pizzella, and F. Ronga (World Scientific, Singapore, 1995).

[2] ROG Collaboration, P. Astone et al., Astropart. Phys. (to be published).

[3] AURIGA Collaboration, M. Cerdonio et al., Gravitational Wave Experiments [1].

[4] G. Frossati, in Graviational Wave Experiments [1].

[5] W. W. Johnson and S. M. Merkowitz, Phys. Rev. Lett. 70, 2367 (1993).

[6] C. Z. Zhou and P. F. Michelson, Phys. Rev. D 51, 2517 (1995)

[7] E. Coccia, J. A. Lobo, and J. A. Ortega, Phys. Rev. D 52, 3735 (1995).

[8] J. A. Lobo, Phys. Rev. D 52, 591 (1995).

[9] R. Forward, Gen. Relativ. Gravit. 2, 149 (1971).

[10] R. V. Wagoner and H. J. Paik, Proceedings of the International Symposium on Experimental Gravitation (Accademia Nazionale dei Lincei, Rome, 1977).

[11] N. S. Magalhaes et al., Mon. Not. R. Astron. Soc. 274, 670 (1995).

[12] J. A. Lobo and M. Serrano, Europhys. Lett. 35, 253 (1996).

[13] A European company fabricating large propellers is LIPS, Drunen (The Netherlands).

[14] A. E. H. Love, A Treatise on the Mathematical Theory of Elasticity (Dover, New York, 1944).

[15] Handbook of Mathematical Functions, edited by M. Abramowitz and I. A. Stegun (Dover, New York, 1972).

[16] R. P. Giffard, Phys. Rev. D 14, 2478 (1976).

[17] G. V. Pallottino and G. Pizzella, Nuovo Cimento C 4, 237 (1981).
[18] K. S. Thorne, in 300 Years of Gravitation, edited by S. W. Hawking and W. Israel (Cambridge University Press, Cambridge, England, 1987).

[19] H. J. Paik, in Gravitational Wave Experiments [1].

[20] W. O. Hamilton, in Proceedings of the 7th Marcel Grossmann Meeting on General Relativity, Stanford, 1994, edited by R. T. Jantzen, G. MacKeiser, and R. Ruffini (World Scientific, Singapore, 1996).

[21] G. Frossati, in Proceedings of the International Conference on Gravitational Waves: Sources and Detectors, Cascina, Italy, 1996, edited by I. Ciufolini and F. Fidecaro (World Scientific, Singapore, 1997).

[22] S. Johnston et al., Nature (London) 361, 613 (1993).

[23] D. Dewey, Phys. Rev. D 36, 1577 (1987).

[24] E. Coccia and V. Fafone, Phys. Lett. A 213, 16 (1996).

[25] S. Vitale et al., in Gravitational Wave Experiments [1].

[26] J. S. Bendat and A. G. Piersol, Measurement and Analysis of Random Data (Wiley, New York, 1966).

[27] P. F. Michelson, Mon. Not. R. Astron. Soc. 227, 933 (1987).

[28] E. E. Flanagan, Phys. Rev. D 48, 2389 (1995).

[29] S. Vitale, M. Cerdonio, E. Coccia, and A. Ortolan, Phys. Rev. D 55, 1741 (1997).

[30] R. Brustein, Gasperini, M. Giovannini, and G. Veneziano, Phys. Lett. B 36, 45 (1995).

[31] P. Astone, J. A. Lobo, and B. F. Schutz, Class. Quantum Grav. 11, 2093 (1994).

[32] VIRGO Collaboration, A. Giazotto et al., in Gravitational Wave Experiments [1].

[33] LIGO team, F. J. Raab, in Gravitational Wave Experiments [1].

[34] G. Frossati and E. Coccia, Cryogenics 34, 9 (1994). 